21st Particles and Nuclei International Conference (PANIC 2017)

International Journal of Modern Physics: Conference Series

Vol. 46 (2018) 1860060 (5 pages)

(C) The Author(s)

DOI: $10.1142 / \mathrm{S} 2010194518600601$

\title{
Study of the Lorentz Structure of the Charged Weak Interaction in Tau Decays at Belle
}

\author{
Junya Sasaki (on behalf of the Belle Collaboration) \\ Department of Physics, University of Tokyo, \\ 7-3-1 Hongo, Bunkyo-ku, Tokyo 113-0033, Japan \\ sasaki@hep.phys.s.u-tokyo.ac.jp
}

Published 3 May 2018

\begin{abstract}
To test the Lorentz structure of the charged weak interaction, we study leptonic decays of tau lepton in the Belle experiment. We perform this test through the measurement of Michel parameters which appear in the differential decay widths of leptonic tau decays. In this paper, we discuss the preliminary results of the measurement of Michel parameters at Belle.
\end{abstract}

Keywords: Tau lepton; Lorentz structure; Michel parameter; weak interaction; Belle experiment.

\section{Introduction}

The Standard Model (SM) is a fundamental theory of elementary particles and their interactions, it combines the electromagnetic and weak interactions into one electroweak interaction through the $\mathrm{U}(1) \times \mathrm{SU}(2)_{L}$ gauge symmetry group. After the symmetry breaking, the electromagnetic interaction remains symmetric under the parity, while the weak interaction exhibits maximal parity violation (maximal asymmetry between left-handed and right-handed fundamental fermions). Leptonic tau decays provide excellent laboratory to study the Lorentz structure of the charged weak current without disturbance from the strong interactions. The most general, Lorentz invariant, derivative-free and lepton-number-conserving four-lepton point interaction matrix element of the $\tau^{-} \rightarrow l^{-} \bar{\nu}_{l} \nu_{\tau}{ }^{\text {a }}$ decay is given by:

$$
\begin{aligned}
& \mathcal{M}=\frac{4 G_{F}}{\sqrt{2}} \sum_{\substack{i=S, V, T \\
j, k=L, R}} g_{j k}^{i}\left[\bar{u}_{j}\left(l^{-}\right) \Gamma^{i} v_{m}\left(\bar{\nu}_{l}\right)\right]\left[\bar{u}_{n}\left(\nu_{\tau}\right) \Gamma_{i} u_{k}\left(\tau^{-}\right)\right], \\
& \Gamma^{S}=1, \Gamma^{V}=\gamma^{\mu}, \Gamma^{T}=\frac{i}{2 \sqrt{2}}\left(\gamma^{\mu} \gamma^{\nu}-\gamma^{\nu} \gamma^{\mu}\right) .
\end{aligned}
$$

\footnotetext{
${ }^{a}$ Unless specified otherwise, charge-conjugated decays are implied throughout the paper.

This is an Open Access article published by World Scientific Publishing Company. It is distributed under the terms of the Creative Commons Attribution 4.0 (CC-BY) License. Further distribution of this work is permitted, provided the original work is properly cited.
} 
Here, $m$ and $n$ are the chiralities of neutrinos and $g_{j k}^{i}$ is dimensionless coupling constant. In the SM, $g_{L L}^{V}=1$ is the only non-zero constant, and $\rho=3 / 4, \eta=0$, $\xi=1, \delta=3 / 4$.

\section{Study of $\tau^{-} \rightarrow l^{-} \nu_{\tau} \bar{\nu}_{l}$}

We measure four Michel parameters $\rho, \eta, \xi$, and $\xi \delta$. The knowledge of tau polarization is necessary to measure the $\xi$ and $\xi \delta$ parameters. While $\rho$ and $\eta$ parameters determine the energy spectrum of the outgoing charged lepton, spin-spin correlations between the $\tau^{+}$and $\tau^{-}$produced at Belle in the $e^{+} e^{-} \rightarrow \tau^{+} \tau^{-}$reaction are exploited to measure also $\xi$ and $\xi \delta$.

Events where signal tau decays via $\tau^{-} \rightarrow \ell^{-} \bar{\nu}_{\ell} \nu_{\tau}$, and the opposite tau decays via $\tau^{+} \rightarrow \pi^{+} \pi^{0} \bar{\nu}_{\tau}$ (or, briefly, $\left(\ell^{-} ; \rho^{+}\right)$) are selected for the analysis. The total differential cross section, $\frac{d \sigma}{d \vec{z}}(\vec{\Theta})$, is linear function on Michel parameters $\vec{\Theta}=\left\{\rho, \eta, \xi_{\rho} \xi, \xi_{\rho} \xi \delta\right\}$. It is used to construct the probability density function (PDF) for the measurement vector $\vec{z}=\left\{p_{\ell}, \cos \theta_{\ell}, \phi_{\ell}, p_{\rho}, \cos \theta_{\rho}, \phi_{\rho}, m_{\pi \pi}, \cos \tilde{\theta}_{\pi}, \tilde{\phi}_{\pi}\right\}$. The Michel parameters are extracted in the unbinned maximum likelihood fit of the $\left(\ell^{-} ; \rho^{+}\right)$events in the full nine-dimensional phase space. The main background processes, $\left(\ell^{-} ; \pi^{+} \pi^{0} \pi^{0}\right),\left(\pi^{-} ; \rho^{+}\right)$and $\left(\rho^{-} ; \rho^{+}\right)$with the fractions, $\lambda_{3 \pi}, \lambda_{\pi}$ and $\lambda_{\rho}$, respectively, are included in the PDF analytically. The remaining background with the fraction $\lambda_{\text {other }}$ is described by the $\mathcal{P}_{\mathrm{bg}}^{\mathrm{MC}}(\vec{z}) \mathrm{PDF}$, which is evaluated from the large Monte Carlo (MC) sample. The total PDF is written as ${ }^{1} 2$ :

$$
\begin{aligned}
& \mathcal{P}(\vec{z})=\frac{\varepsilon(\vec{z})}{\bar{\varepsilon}}\left(\left(1-\lambda_{3 \pi}-\lambda_{\pi}-\lambda_{\rho}-\lambda_{\text {other }}\right) \frac{S(\vec{z} \mid \vec{\Theta})}{\int \frac{\varepsilon(\vec{z})}{\bar{\varepsilon}} S(\vec{z} \mid \vec{\Theta}) d \vec{z}}+\right. \\
& \left.+\lambda_{3 \pi} \frac{B_{3 \pi}(\vec{z} \mid \vec{\Theta})}{\int \frac{\varepsilon(\vec{z})}{\bar{\varepsilon}} B_{3 \pi}(\vec{z} \mid \vec{\Theta}) d \vec{z}}+\lambda_{\pi} \frac{B_{\pi}(\vec{z})}{\int \frac{\varepsilon(\vec{z})}{\bar{\varepsilon}} B_{\pi}(\vec{z}) d \vec{z}}+\lambda_{\rho} \frac{B_{\rho}(\vec{z})}{\int \frac{\varepsilon(\vec{z})}{\bar{\varepsilon}} B_{\rho}(\vec{z}) d \vec{z}}+\lambda_{\text {other }} \mathcal{P}_{\mathrm{bg}}^{\mathrm{MC}}(\vec{z})\right),
\end{aligned}
$$

where $S(\vec{z} \mid \vec{\Theta}), \quad B_{3 \pi}(\vec{z} \mid \vec{\Theta}), \quad B_{\pi}(\vec{z})$ and $B_{\rho}(\vec{z})$ are the cross sections for the $\left(\ell^{-} ; \rho^{+}\right), \quad\left(\ell^{-} ; \pi^{+} \pi^{0} \pi^{0}\right), \quad\left(\pi^{-} ; \rho^{+}\right)$and $\left(\rho^{-} ; \rho^{+}\right)$events, respectively; $\varepsilon(\vec{z})$ is the detection efficiency for signal events in the full phase space; and $\bar{\varepsilon}=$ $\int \varepsilon(\vec{z}) S\left(\vec{z} \mid \vec{\Theta}_{\mathrm{SM}}\right) d \vec{z} / \int S\left(\vec{z} \mid \vec{\Theta}_{\mathrm{SM}}\right) d \vec{z}$ is an average signal detection efficiency. The $\xi_{\rho}$ is determined from the study of the $\left(\tau^{-} \rightarrow \rho^{-} \nu_{\tau} ; \tau^{+} \rightarrow \rho^{+} \bar{\nu}_{\tau}\right)$ events.

The uncertainties arising from the physical and apparatus corrections to the $\mathrm{PDF}$, as well as from the normalization are well below $1 \%$, however we still observe a systematic bias of the order of a few percent, especially in the $\xi_{\rho} \xi$ and $\xi_{\rho} \xi \delta$ parameters, coming from the description of the experimental trigger efficiency corrections.

\section{Study of $\tau^{-} \rightarrow l^{-} \nu_{\tau} \bar{\nu}_{l} \gamma$}

Emission of the photon in the final state results in three additional parameters: $\bar{\eta}, \eta^{\prime \prime}$ and $\xi \kappa$. The $\xi \kappa$, like $\xi$ and $\delta$, appears in the tau spin-dependent part of 
the differential decay width, so, spin-spin correlations between the $\tau^{+}$and $\tau^{-}$ allow one to measure $\xi \kappa$. In this analysis, $\rho, \eta, \xi, \delta$ and $\xi_{\rho}$ parameters are fixed to their SM values. Also, feasibility study showed that the sensitivity to the $\eta^{\prime \prime}$ is very poor in comparison with the $\bar{\eta}$ and $\kappa$, so, $\eta^{\prime \prime}$ was also fixed to its $\mathrm{SM}$ expectation $\eta^{\prime \prime}=0$. In the signal events one tau decays to radiative leptonic mode, $\tau^{-} \rightarrow \ell^{-} \bar{\nu}_{\ell} \nu_{\tau} \gamma$, while the other tau decays via $\tau^{+} \rightarrow \pi^{+} \pi^{0} \bar{\nu}_{\tau}$ (or, shortly, $\left.\left(\ell^{-} \gamma ; \rho^{+}\right)\right)$. The PDF function for the vector of the measured parameters $\vec{z}=\left\{p_{\ell}, \cos \theta_{\ell}, \phi_{\ell}, p_{\gamma}, \cos \theta_{\gamma}, \phi_{\gamma}, p_{\rho}, \cos \theta_{\rho}, \phi_{\rho}, m_{\pi \pi}, \cos \tilde{\theta}_{\pi}, \tilde{\phi}_{\pi}\right\}$ is constructed from the total differential cross section, $\frac{d \sigma}{d \vec{z}}(\vec{\Theta})$, which is linear function on $\vec{\Theta}=$ $\{\bar{\eta}, \xi \kappa\},\left(\vec{\Theta}_{\mathrm{SM}}=\{0,0\}\right)$. Michel parameters are extracted in the unbinned maximum likelihood fit of the $\left(\ell^{-} \gamma ; \rho^{+}\right)$events in the full twelve-dimensional phase space. The dominant background contributions are included in the PDF analytically, while the remaining background with the fraction $\lambda_{0}$ is described by the $\mathcal{P}_{\mathrm{bg}}^{\mathrm{MC}}(\vec{z}) \mathrm{PDF}$, evaluated from the MC sample. The total PDF is written as:

$$
\mathcal{P}(\vec{z})=\left(1-\sum_{i=0}^{2(5)} \lambda_{i}\right) \frac{S(\vec{z} \mid \vec{\Theta}) \varepsilon(\vec{z})}{\int \mathrm{d} \vec{z} S(\vec{z} \mid \vec{\Theta}) \varepsilon(\vec{z})}+\sum_{i=1}^{2(5)}\left(\lambda_{i} \frac{B_{i}(\vec{z}) \varepsilon(\vec{z})}{\int \mathrm{d} \vec{z} B_{i}(\vec{z}) \varepsilon(\vec{z})}\right)+\lambda_{0} \mathcal{P}_{\mathrm{bg}}^{\mathrm{MC}}(\vec{z}),
$$

where $S(\vec{z} \mid \vec{\Theta})$ is analytical cross section for the signal, $B_{i}(\vec{z})$ is analytical cross section of the $i$ th background $\left(i=1 \div 2\right.$ for the $\left(e^{-} \gamma ; \rho^{+}\right)$, and $i=1 \div 5$ for the ( $\left.\mu^{-} \gamma ; \rho^{+}\right)$events), $\lambda_{i}$ is the fraction of the $i$ th background and $\varepsilon(\vec{z})$ is the detection efficiency in the full phase space.

Figure 1 shows the distribution of the signal photon energy for the $\left(e^{-} \gamma ; \rho^{+}\right)$ and $\left(\mu^{-} \gamma ; \rho^{+}\right)$events, as well as the contour of the likelihood function on the $\bar{\eta}-\xi \kappa$ plane ${ }^{3}$. The Belle preliminary result is ${ }^{4}$ :

$$
\begin{gathered}
\bar{\eta}=-1.3 \pm 1.5 \pm 0.8 \\
\xi \kappa=0.5 \pm 0.4 \pm 0.2
\end{gathered}
$$

The $\bar{\eta}$ and $\xi \kappa$ are consistent with their SM expectations within uncertainties.
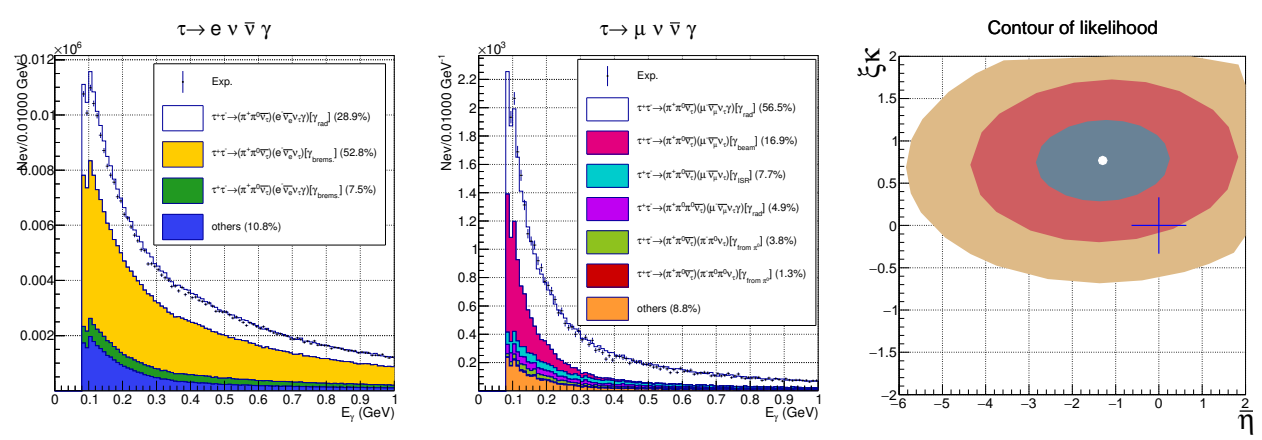

Fig. 1. The distribution of the signal photon energy for the $\left(e^{-} \gamma ; \rho^{+}\right)$(left) and $\left(\mu^{-} \gamma ; \rho^{+}\right)$ (middle) events. The contours of the likelihood function on the $\bar{\eta}-\xi \kappa$ plane (right), the SM prediction is shown by the cross. 


\section{J. Sasaki}

Table 1. Branching fractions of five-body leptonic decays predicted by the SM, and expected uncertainties at Belle.

\begin{tabular}{ccc}
\hline Mode & Predicted $B R$ from Ref. ${ }^{6}$ & Expected Total Error $\left(B R_{\exp }\right)$ \\
\hline$\tau^{-} \rightarrow e^{-} e^{+} e^{-} \bar{\nu}_{e} \nu_{\tau}$ & $(4.21 \pm 0.01) \times 10^{-5}$ & $10 \%$ \\
$\tau^{-} \rightarrow \mu^{-} e^{+} e^{-} \bar{\nu}_{\mu} \nu_{\tau}$ & $(1.984 \pm 0.004) \times 10^{-5}$ & $15 \%$ \\
$\tau^{-} \rightarrow e^{-} \mu^{+} \mu^{-} \bar{\nu}_{e} \nu_{\tau}$ & $(1.247 \pm 0.001) \times 10^{-7}$ & $70 \%$ \\
$\tau^{-} \rightarrow \mu^{-} \mu^{+} \mu^{-} \bar{\nu}_{\mu} \nu_{\tau}$ & $(1.183 \pm 0.001) \times 10^{-7}$ & $70 \%$ \\
\hline
\end{tabular}

\section{Study of $\tau^{-} \rightarrow l^{-} l^{+} l^{\prime-} \nu_{\tau} \bar{\nu}_{l}$}

In this study, we set constraints on Michel parameters, $Q_{R L}, Q_{L R}, Q_{R R}, B_{R L}$, $B_{L R}, I_{\alpha}$, and $I_{\beta}$, from the measurement of the branching fractions of the five-body leptonic decays. The SM predicts that only $Q_{L L}=1$ has a non-zero value ${ }^{5}$. The theoretical formula for the branching fraction $(B R)$ can be written as:

$$
B R_{\exp }=B R_{\mathrm{SM}}\left[Q_{L L}+b Q_{L R}+c B_{L R}+Q_{R R}+d Q_{R L}+e B_{R L}+\Re(f) I_{\alpha}+\Re(g) I_{\beta}\right] .
$$

Here, $B R_{\exp }$ is experimentally measured branching fraction, $B R_{\mathrm{SM}}$ is the branching fraction predicted in the SM (see Table 1), Actually, the coefficients $b, c, d$, and $e$ have a relation $b=d, c=e^{6}$. The difference between the SM-prediction and the experimentally measured value, $\delta(B R)\left(B R_{\exp }=B R_{\mathrm{SM}}+\delta(B R)\right)$,

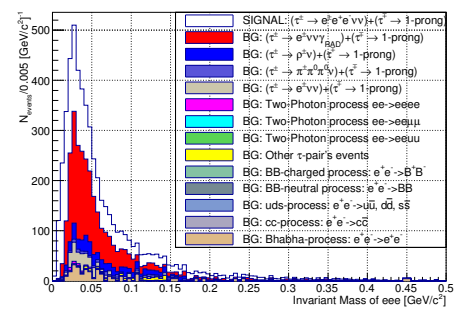

(a)

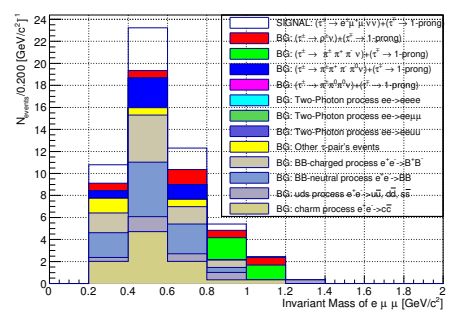

(c)

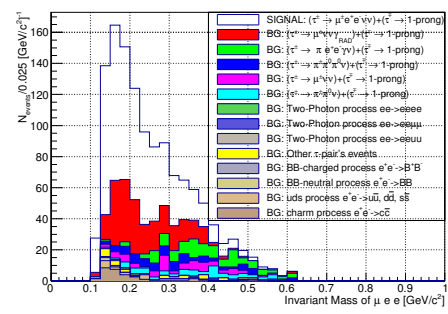

(b)

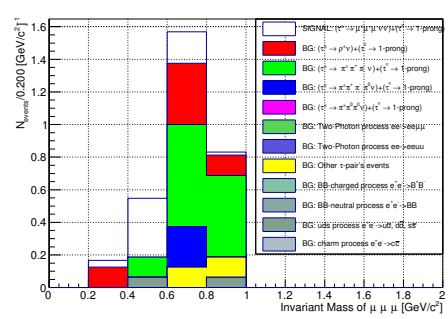

(d)

Fig. 2. Distribution of the invariant mass of three charged outgoing leptons, $M_{l l^{\prime} l^{\prime}}$, for the selected events: (a) $\tau^{-} \rightarrow e^{-} e^{+} e^{-} \bar{\nu}_{e} \nu_{\tau}$, (b) $\tau^{-} \rightarrow \mu^{-} e^{+} e^{-} \bar{\nu}_{\mu} \nu_{\tau}$, (c) $\tau^{-} \rightarrow e^{-} \mu^{+} \mu^{-} \bar{\nu}_{e} \nu_{\tau}$, and (d) $\tau^{-} \rightarrow$ $\mu^{-} \mu^{+} \mu^{-} \bar{\nu}_{\mu} \nu_{\tau}$. Open histograms show signal events, shaded histograms - background contributions. 
allows us to constrain Michel parameters. We estimated the expected uncertainties (statistical $\oplus$ systematic) of the $B R_{\exp }$ at Belle, they are shown in Table 1.

In our study, the main background for the $\tau^{-} \rightarrow e^{-} e^{+} e^{-} \bar{\nu}_{e} \nu_{\tau}$ and $\tau^{-} \rightarrow$ $\mu^{-} e^{+} e^{-} \bar{\nu}_{\mu} \nu_{\tau}$ modes comes from the radiative leptonic tau decay, $\tau^{-} \rightarrow l^{-} \bar{\nu}_{l} \nu_{\tau} \gamma$, due to $\gamma$-conversion $\left(\gamma \rightarrow e^{+} e^{-}\right)$in the material of the detector. The hadronic tau decays, such as $\tau^{-} \rightarrow \rho^{-} \nu_{\tau}$ and $\tau^{-} \rightarrow a_{1}^{-} \nu_{\tau}$, contaminate all four modes due to their large branching fractions and misidentification of charged pion as electron or muon. For the case of $\tau^{-} \rightarrow e^{-} \mu^{+} \mu^{-} \bar{\nu}_{e} \nu_{\tau}$, the main background comes from non-tau pair processes. The main background to the $\tau^{-} \rightarrow \mu^{-} \mu^{+} \mu^{-} \bar{\nu}_{\mu} \nu_{\tau}$ decay comes mostly from the other tau decays. The measurement of the branching fractions is performed as a blinded analysis. Figure 2 shows the expected background contamination from the MC simulation.

\section{Conclusion}

Study of the Lorentz structure of the charged weak interaction in leptonic tau decays at Belle is ongoing.

\section{References}

1. D. A. Epifanov [Belle Collaboration], Nucl. Part. Phys. Proc. 287-288, 7 (2017).

2. D. A. Epifanov [Belle Collaboration], Slide of The International Workshop on $e^{+} e^{-}$ collisions from Phi to Psi 2017, "Experimental review of tau lepton studies at the B factories"

3. N. Shimizu [Belle Collaboration], Nucl. Part. Phys. Proc. 287-288, 11 (2017).

4. N. Shimizu et al. (Belle Collaboration), Measurement of the tau Michel parameters $\bar{\eta}$ and $\xi \kappa$ in the radiative leptonic decay $\tau^{-} \rightarrow l^{-} \nu_{\tau} \bar{\nu}_{l} \gamma$, [arXiv:1709.08833v2 [hep-ex]] (2017)

5. W. Fetscher, H. J. Gerber and K. F. Johnson, Phys. Lett. B 173, 102 (1986).

6. A. Flores-Tlalpa, G. Lopez Castro and P. Roig, JHEP 1604, 185 (2016) [arXiv:1508.01822 [hep-ph]]. 